\title{
Transferrin gene polymorphisms alter the transferrin focusing pattern, making congenital disorder of glycosylation diagnosis difficult
}

\author{
Patryk Lipiński ${ }^{1}$ Anna Bogdańska², Agnieszka Sobczyńska-Tomaszewska³ and \\ Anna Tylki-Szymańska ${ }^{1}$
}

1Department of Pediatrics, Nutrition and Metabolic Diseases, The Children's Memorial Health Institute, Warsaw, Poland; ${ }^{2}$ Department of Biochemistry, Radioimmunology and Experimental Medicine, The Children's Memorial Health Institute, Warsaw, Poland; ${ }^{3}$ MedGen Medical Centre, Warsaw, Poland

Background: Several transferrin gene polymorphisms are known to result in a shifted IEF pattern. The aim of this study was to characterize the transferrin gene polymorphisms observed in patients from one referral center. Materials and methods: Patients with solely increased pentasialo-Tf were selected. The whole exome sequencing was done from probands (patients) and from DNA available from their parents. Results: Two various polymorphisms in the transferrin gene: c.2012G>A, p.Gly671Glu and c.1027C $>$ T, p.Arg343Trp, were found. Conclusions: Two transferrin gene polymorphisms: c.2012G >A, p.(Gly671Glu) and c.1027C >T, p.(Arg343Trp) solely correspond to an elevated pentasialo-Tf.

Keywords: transferrin; congenital disorders of glycosylation; polymorphism; isoelectric focusing; whole-exome sequencing

Received: 26 May, 2021; revised: 28 June, 2021; accepted: 03 August, 2021; available on-line: 06 September, 2021

छe-mail: p.lipinski@ipczd.pl

Funding sources: This study was funded by the Children's Memorial Health Institute intramural grant S190/2020.

Abbreviations: CDG, congenital disorders of glycosylation; CNV, copy number variations; IEF, isoelectric focusing; MS, mass spectrometry; SNV, single nucleotide variants; Tf, transferrin; WES, whole exome sequencing

\section{BACKGROUND}

Congenital disorders of glycosylation (CDG) comprise a group of rare inherited metabolic disorders caused by a deficient glycosylation of proteins and lipids (Jaeken et al., 2017; Ferreira et al., 2018; Peanne et al., 2018; Francisco et al., 2019). Isoelectric focusing (IEF) of serum transferrin ( $\mathrm{Tf}$ ) is the method of choice for diagnosis of N-glycosylation disorders associated with sialic acid deficiency (Jaeken et al., 2017; Ferreira et al., 2018; Peanne et al., 2018; Francisco et al., 2019). Based on the Tf isoform profile, patients can be classified into CDG type I, CDG type II or a mixed CDG type I/II. Type 1 pattern (CDG-I) is associated with an increased asialo-, disialo-Tf, and decreased tetrasialo-Tf; it indicates an assembly or transfer defect of the dolichol-linked glycan (Lefeber et al., 2011). Type 2 pattern (CDG-II) indicates a processing defect during Golgi glycosylation; an increased asialo-, monosialo-, disialo- and trisialo-Tf are observed there (Lefeber et al., 2011).

Several Tf gene polymorphisms are known to result in a shifted IEF pattern, caused by pI differences of the polypeptide chain (Lefeber et al., 2011; Jaeken et al.,
2017; Ferreira et al., 2018; Peanne et al., 2018; Francisco et al., 2019). To improve diagnostics of CDG, mass spectrometry (MS) methods for glycoprotein analysis were developed, however, they are not routinely available (Guillard et al., 2011; Van Scherpenzeel et al., 2016).

The aim of this study was to characterize transferrin gene polymorphisms observed in patients screened for CDG in one referral center, to evaluate their overall prevalence and facilitate interpretation of laboratory findings.

\section{MATERIALS AND METHODS}

\section{Serum transferrin (Tf) isoforms isoelectrofocusing}

Serum Tf isoforms were analyzed by isoelectrofocusing (IEF) agarose gel electrophoresis according to the modification introduced in our laboratory (Adamowicz et al., 1996; Adamowicz et al., 2007) of the method described by Van Eijk and others (Van Eijk et al., 1982). Since 1995, the CDG selective screening based on serum Tf IEF has been performed at our Institute (CMHI) for patients from the entire country (Bogdańska et al., 2021; Lipiński et al., 2021). During the years of 1995-2020, a total number of 23,183 serum Tf isoform analyses from pediatric patients have been performed. Out of all screened patients, patients were observed with solely increased pentasialo-Tf and patients with solely increased trisialo-Tf, corresponding to Tf gene polymorphisms. The prevalence of those polymorphisms was estimated. The clinical phenotype of patients with $\mathrm{Tf}$ gene polymorphisms was not significant.

\section{Molecular analysis}

Secondly, in the group of pediatric patients (probands, $n=4)$ and their parents $(n=4)$ in whom the same IEF pattern was observed and DNA was available, molecular analysis was performed. Whole exome sequencing (WES) was done to search for single nucleotide variants (SNV) and copy number variations $(\mathrm{CNV})$. The patients' genomic DNA was extracted from the whole blood sample, and the sequencing library was prepared according to the Agilent Sure-Select Human All Exon V5 protocol. Enriched DNA libraries were sequenced by the Illumina HiSeq4000 instrument. The average depth of coverage for the TF gene was $100 \%$ per $30 \times$. 
Table 1. Serum Tf isoforms percentage (\%) in patients with Tf gene polymorphism.

\begin{tabular}{lll}
\hline Tf isoform & $\begin{array}{l}\text { Tf isoform \% in pa- } \\
\text { tients with Tf gene } \\
\text { polymorphism }\end{array}$ & $\begin{array}{l}\text { Reference ranges for Tf } \\
\text { isoform \% }\end{array}$ \\
\hline Asialo- & 0 & 0 \\
\hline Monosialo- & 0 & 0 \\
\hline Disialo- & $3.3-4.3$ & $1.5-6.2$ \\
\hline Trisialo- & $10.1-13.6$ & $7.4-17.1$ \\
\hline Tetrasialo- & $33.6-36.8$ & $55.7-67.2$ \\
\hline Pentasialo- & $32.4-37.8$ & $13.2-19.9$ \\
\hline
\end{tabular}

The presence of the variant in control populations was verified at 1000Genomes (http://www. 1000genomes. org), the Exome Variant Server (http://evs. gs.washington.edu/EVS), the Exome Aggregation Consortium (http://exac.broadinstitute.org), gnomAD (http://gnomad.broadinstitute.org/) and MedGen laboratory database that contained records of subjects with the Polish origin.

This study has been approved by an ethics committee of The Children's Memorial Health Institute in Warsaw, Number 23/KBE/2020. Informed consent was obtained from all included patients.

\section{RESULTS}

\section{Serum transferrin (Tf) isoforms' isoelectrofocusing}

In this study, a total number of 55 pediatric patients with solely increased pentasialo-Tf was identified; the prevalence of this phenomenon was estimated as $0.2 \%$. A solely increased trisialo-Tf was identified in 15 pediatric patients; the prevalence of this phenomenon was estimated as $0.06 \%$.

In the group of 4 children (probands) with solely increased pentasialo-Tf (Table 1) and their parents $(n=4)$ with the same serum Tf IEF profile, from whom DNA was available, molecular analysis was performed. A graphic presentation (IEF profiles) of the serum Tf gene polymorphism resulting in solely increased pentasialo-Tf is presented in Fig. 1.

\section{Whole exome sequencing}

In the study group, we identified two polymorphisms in the Tf gene in a heterozygous state: c.2012G $>$ A, p.Gly671Glu (3 probands) and c.1027C $>$ T, p.Arg343Trp (1 proband). A detailed characteristic of those two variants is presented in $\mathrm{Ta}$ ble 2 .

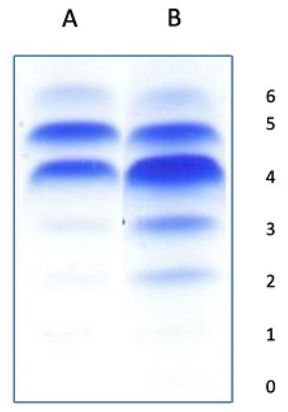

Figure 1. IEF of serum transferrin gene polymorphism resulting in increased pentasialo-Tf solely.

(A) transferrin gene polymorphism (solely increased pentasialoTf); (B) normal profile; 0, asialo-Tf; 1, monosialo-Tf; 2, disialo-Tf; 3, trisialo-Tf; 4, tetrasialo-Tf; 5, pentasialo-Tf; 6, hexasialo-Tf.

\section{DISCUSSION}

Transferrin ( $\mathrm{Tf}$ ) is a plasma iron transport protein, with two asparagine N-glycosylation sites (Asn432 and Asn630). The glycans are each terminated by two negatively charged molecules of sialic acid that allow discrimination using IEF (Lefeber et al., 2011). The dominant isoform in healthy individuals is tetrasialo-Tf, while pentasialo-, hexasialo-, trisialo- and disialo-Tf occur in much smaller amounts (Lefeber et al., 2011).

In the study presented here we identified two transferrin gene polymorphisms: c.2012G>A, p.(Gly671Glu) and c.1027C $>$ T, p.(Arg343Trp) corresponding to solely elevated pentasialo-Tf IEF pattern.

So far, the c.2012G>A, p.(Gly671Glu) transferrin polymorphism, also known as transferrin B2, represents the best known (De Wolf et al., 2011; Park et al., 2014; Zühlsdorf et al., 2015). The observed IEF pattern could be explained by an amino acid change from an uncharged side-chain of glycine to the acid side-chain of glutamic acid, which adds a negative charge.

The second variant identified in our study, c. $1027 \mathrm{C}>\mathrm{T}$, p.(Arg343Trp), was previously described by Zühlsdorf and others (Zühlsdorf et al., 2015). The observed IEF pattern could be explained by an amino acid change from the positively charged side chain of arginine to the uncharged side chain of tryptophan.

Park and others (Park et al., 2014) have recently described another variant, c.1775A $>$ C, p.(GluE592Ala), resulting in an increased pentasialo-Tf fraction. There are also other transferrin variants, like c.1765C $>\mathrm{T}$, p.(Pro589Ser), also known as Transferrin C2, or c.829G $>$ A, p.(Gly277Ser) resulting in an increased trisialo-Tf fraction (De Wolf et al., 2011; Kutalik et al., 2011).

In the diagnosis of CDG, it is important to exclude secondary causes of N-hypoglycosylation, as well as transferrin gene polymorphisms (Jaeken et al., 2017;

Table 2. Characteristics of Tf gene polymorphisms.

\begin{tabular}{|c|c|c|}
\hline Variant details & rs121918677 & rs150854910 \\
\hline Variant type & single nucleotide variant & single nucleotide variant \\
\hline HGVS & NM_001063.4:c.2012G>A & NM_001063.4:c.1027C>T \\
\hline Protein change & p.Gly671Glu & p.Arg343Trp \\
\hline Molecular consequence & Missense variant & Missense variant \\
\hline Clinical significance & Transferrin variant B2 & not reported in ClinVar \\
\hline References & $\begin{array}{l}\text { Zühlsdorf A et al., 2015; Park JH et al., 2014; de Wolf } \\
\text { HK et al., 2011; Kutalik Z et al., } 2011\end{array}$ & Zühlsdorf A et al., 2015 \\
\hline
\end{tabular}


Ferreira et al., 2018; Peanne et al., 2018; Francisco et al., 2019). All described polymorphisms change the charge of transferrin, affecting the IEF pattern. This fact can impose some diagnostic difficulties in patients suspected of CDG. The implementation of MS was reported to be helpful in identification of those polymorphisms (Guillard et al., 2011; Van Scherpenzeel et al., 2016). However, these high-throughput screening technologies are not routinely available. Since the next-generation sequencing became more widely available, molecular analyses comprise an alternative method for transferrin polymorphism identification.

A major limitation of our study is a small number of patients who underwent molecular analyses. The second one is the fact that serum Tf isoforms were analyzed only by IEF. Thus, an MS study will be essential to reveal changes in charge of the transferrin molecules.

\section{CONCLUSIONS}

1. Two transferrin gene polymorphisms: c.2012G>A, p.(Gly671Glu) and c.1027C $>$ T, p.(Arg343Trp) correspond to solely elevated pentasialo-Tf IEF pattern.

2. Whole exome sequencing represents a useful tool for identifying transferrin gene polymorphisms.

\section{Authors' contributions}

Project administration: Patryk Lipiński, Anna TylkiSzymańska; Investigation: Patryk Lipiński, Anna Bogdańska, Agnieszka Sobczyńska-Tomaszewska, Anna Tylki-Szymańska Supervision: Anna Tylki-Szymańska; Writing - original draft: Patryk Lipiński; Writing - review and editing: Patryk Lipiński, Anna Tylki-Szymańska.

\section{Competing interests}

All authors certify that they have NO affiliations with or involvement in any organization or entity with any financial or non-financial interest in the subject matter or materials discussed in this manuscript.

\section{REFERENCES}

Adamowicz M, Pronicka E (1996) Carbohydrate deficient glycoprotein syndrome-like transferrin isoelectric focusing pattern in untreated fructosaemia. Eur J Pediatr 155: 347-348. https://doi.org/0.1007/ BF02002730

Adamowicz M, Płoski R, Rokicki D, Morava E, Gizewska M, Mierzewska H, Pollak A, Lefeber DJ, Wevers RA, Pronicka E (2007) Transferrin hypoglycosylation in hereditary fructose intolerance: us- ing the clues and avoiding the pitfalls. I Inherit Metab Dis 30: 407. https://doi.org/10.1007/s10545-007-0569-z

Bogdańska A, Lipiński P, Szymańska-Rożek P, Jezela-Stanek A, Rokicki D, Socha P, Tylki-Szymańska A (2021) Clinical, biochemical and molecular phenotype of congenital disorders of glycosylation: longterm follow-up. Orphanet J Rare Dis 16: 17. https://doi.org/10.1186/ s13023-020-01657-5

De Wolf HK, Huijben K, van Wijnen M, de Metz M, Wielders JP (2011) A novel C2 transferrin variant interfering with the analysis of carbohydrate-deficient transferrin. Clin Chim Acta 412: 1683-1685. https://doi.org/10.1016/j.cca.2011.05.008

Ferreira CR, Altassan R, Marques-Da-Silva D, Francisco R, Jaeken J, Morava E (2018) Recognizable phenotypes in CDG. J Inherit Metab Dis 41: 541-553. https://doi.org/10.1007/s10545-018-0156-5

Francisco R, Marques-da-Silva D, Brasil S, Pascoal C, Dos Reis Ferreira V, Morava E, Jaeken J (2019) The challenge of CDG diagnosis. Mol Genet Metab 126: 1-5. https://doi.org/10.1016/j.ymgme.2018.11.003

Guillard M, Wada Y, Hansikova H, Yuasa I, Vesela K, Ondruskova N, Kadoya M, Janssen A, Van den Heuvel LP, Morava E, Zeman J, Wevers RA, Lefeber DJ (2011) Transferrin mutations at the glycosylation site complicate diagnosis of congenital disorders of glycosylation type I. J Inherit Metab Dis 34: 901-906. https://doi. org/10.1007/s10545-011-9311-y

Jaeken J, Peanne R (2017) What is new in CDG? J Inberit Metab Dis 40: 569-586. https://doi.org/10.1007/s10545-017-0050-6

Kutalik Z, Benyamin B, Bergmann S, Mooser V, Waeber G, Montgomery GW, Martin NG, Madden PA, Heath AC, Beckmann JS, Vollenweider P, Marques-Vidal P, Whitfield JB (2011) Genome-wide association study identifies two loci strongly affecting transferrin glycosylation. Hum Mol Genet 20: 3710-3717. https://doi. org $/ 10.1093 / \mathrm{hmg} / \mathrm{ddr} 272$

Lefeber DJ, Morava E, Jaeken J (2011) How to find and diagnose a CDG due to defective N-glycosylation. I Inherit Metab Dis 34: 849852. https://doi.org/10.1007/s10545-011-9370-0

Lipiński P, Bogdańska A, Tylki-Szymańska A (2021) Congenital disorders of glycosylation: Prevalence, incidence and mutational spectrum in the Polish population. Mol Genet Metab Rep 27: 100726. https:// doi.org/10.1016/j.ymgmr.2021.100726

Park JH, Zühlsdorf A, Wada Y, Roll C, Rust S, Du Chesne I, Grüneberg M, Reunert J, Marquardt T (2014) The novel transferrin E592A variant impairs the diagnostics of congenital disorders of glycosylation. Clin Chim Acta 436: 135-139. https://doi.org/10.1016/j. cca.2014.05.011

Péanne R, de Lonlay P, Foulquier F, Kornak U, Lefeber DJ, Morava E, Pérez B, Seta N, Thiel C, Van Schaftingen E, Matthijs G, Jaeken J (2018) Congenital disorders of glycosylation (CDG): Quo vadis? Eur J Med Genet 61: 643-663. https://doi.org/10.1016/j. ejmg.2017.10.012

Van Eijk HG, van Noort WL, Kros MJ, van der Huel C (1982) The heterogeneity of human serum transferrin and human transferrin preparations on isoelectric focusing gels: no functional difference of the fraction in vitro. Clin Chim Acta 121: 209-216. https://doi. org/10.1016/0009-8981(82)90060-2

Van Scherpenzeel M, Willems E, Lefeber DJ (2016) Clinical diagnostics and therapy monitoring in the congenital disorders of glycosylation. Glycoconj I 33: 345-358. https://doi.org/10.1007/s10719-015-9639-x

Zühlsdorf A, Park JH, Wada Y, Rust S, Reunert J, DuChesne I, Grüneberg M, Marquardt T (2015) Transferrin variants: pitfalls in the diagnostics of Congenital disorders of glycosylation. Clin Biochem 48: 11-43. https://doi.org/10.1016/j.clinbiochem.2014.09.022 